\title{
Green nanotechnology in cadmium sulphide nanoparticles and understanding its toxicity and antimicrobial properties.
}

\author{
Padmini V Sekar, V Deepa Parvathi, Sumitha R* $^{*}$ \\ Department of Biomedical Sciences, Sri Ramachandra Institute of Higher Education and Research, Chennai, India
}

\begin{abstract}
Nano scale materials possess unique properties that are generally not seen in their bulk counterparts. Though chemical and physical methods of nano particle synthesis is widely used, the advantage of biological synthesis have gained popularity owing to their environment affability. Green synthesis of nanoparticles has gained importance in biomedical research owing to its ease of synthesis, better stability of the nanoparticles and cost effectiveness. Cadmium sulphide nanoparticles (CdS) have been extensively studied to have potential applications in the field of biomedical research and imaging studies.In the present study, CdS nanoparticles were synthesized using Bacillus licheniformis as the precursor. The thus synthesized CdS nanoparticles were characterized by scanning electron microscopy and the nano dimension of the particle was confirmed. Four different concentrations of CdS nanoparticles were tested to assess its genotoxicity using Drosophila melanogaster as the in-vivo model. Additionally, the antimicrobial property of $\mathrm{CdS}$ was also examined by well diffusion and macro dilution method. The results suggested significant antimicrobial activity and lack of genotoxicity of CdS nanoparticles.
\end{abstract}

Keywords: Cadmium sulphide (CdS) nanoparticles, Green synthesis, Drosophila melanogaster, Antimicrobial activity.

Accepted on July 24, 2019

\section{Introduction}

Nanotechnology is a promising field of science with immense scope in the field of medicine. Biological synthesis of nanomaterials through natural sources is an emerging approach in the field of nanomedicine. Many techniques are available to synthesize diverse categories of nanoparticles integrating physical, chemical, biological and hybrid methods [1-4]. Exploitation of toxic chemicals for the synthesis of nanoparticles has limited its application in the field of medicine. The use of natural precursors for the synthesis of nanomaterial has opened new avenues in the field of nanobiotechnology. Nanoparticle production using microbial forms has a significant prospective to support nanoparticles synthesis that does not require any of the toxic and hazardous, chemicals that are routinely used [5]. Cadmium sulphide nanoparticles $(\mathrm{CdS})$ was chosen as a compound of interest due to its high stability, excellent physical, chemical and structural properties, ease of preparation and handling. CdS nanoparticles are being used widely used for the cancer diagnosis and antimicrobial treatment [6,7]. CdS nanoparticles have proven antimicrobial properties against both gram-positive and negative showing broad spectrum antibacterial potential [8]. However, assessment of their toxicity is extremely important for significant applications in medicine.

\section{Materials and Methods}

\section{Culture initiation}

Bacillus licheniformis (ATCC 12759) strain was purchased from Himedia, Chennai, India. The bacterial strain was inoculated on nutrient broth. The isolate was maintained in nutrient agar for further study [9].

\section{Microbial synthesis of cadmium sulphide nanoparticles}

The initiated culture was centrifuged at $8000 \mathrm{rpm}$ for 15 minutes. The pellet was washed repeatedly to remove remnant media. The biomass was carefully weighed and deionized water was added. This set up (in an Erlenmeyer flask) was maintained in a shaker for 30 minutes at $200 \mathrm{rpm} .0 .1 \mathrm{mM}$ of cadmium chloride and $0.01 \mathrm{mM}$ of sodium sulphide was added to the mixture. Post incubation, the contaminants were excluded using acetone and water to obtain pure nanoparticles. The precipitate was lyophilized. 


\section{Characterization of cadmium sulphide nanoparticles using scanning electron microscopy}

The lyophilized sample was characterized by Scanning Electron Microscopy. The SEM analysis was carried out at Anna University, Chennai.

\section{In vivo toxicity assessment on Drosophila melanogaster}

Phenotypic analysis: In vivo toxicity assessment was done using Drosophila melanogaster as a model organism. Flies were bred and maintained in the laboratory using corn meal agar medium. The flies were exposed to four different concentrations of the synthesized CdS nanoparticles (20, 40, 60 and $80 \mu \mathrm{g} / \mathrm{ml}$ ) for 24 and 48 hours respectively. Benzaldehyde was used as the positive control and water was used as negative control. Post exposure, phenotypic changes were analyzed using stereo zoom microscope (Nikon-Model CDSS230) and documented [10].

DNA fragmentation assay: 50 flies were exposed to four different concentrations of the synthesized $\mathrm{CdS}$ nanoparticles (20, 40, 60 and $80 \mu \mathrm{g} / \mathrm{ml})$ along with positive and negative control. Post exposure of DNA from exposed flies was extracted by phenol-chloroform method to evaluate the genotoxic effect of CdS nanoparticles.

Quantification of DNA by Nanodrop: The isolated DNA of the exposed flies was quantified using Nanodrop ND-1000 Spectrophotometer. Quantity and quality of the DNA were measured by reading the whole absorption spectrum and calculating the concentration and absorbance ratio at 260/280 and $230 / 260 \mathrm{~nm}$ respectively.

Preparation of the inoculum for antimicrobial sensitivity tests: The culture initiation was done by inoculating the fresh nutrient broth with the strain and incubating it overnight at $37^{\circ}$ C. Using McFarland's turbidity as the standard, the inoculum was prepared $(5 \times 105 \mathrm{CFU} / \mathrm{ml})$.

Antimicrobial sensitivity test by well diffusion method: Antibacterial activity of $\mathrm{CdS}$ nanoparticles was done by agar well diffusion method on Escherichia coli and Staphylococcus aureus. Preparation of the inoculum containing $106 \mathrm{cfu} / \mathrm{ml}$ of each bacterial culture to be tested was spread on nutrient agar plates using moistened sterile swab with the bacterial suspension. $8 \mathrm{~mm}$ wells were punched and the defined concentrations of $\mathrm{CdS}$ nanoparticles were added. The antimicrobial effect was compared with commercially available antibiotics ciprofloxacin and amoxicillin. The zone of clearance obtained after incubation period of 24 hours was noted [11].

Antimicrobial susceptibility test by macro dilution method: The antibiotic susceptibility test was done against Escherichia coli and Staphylococcus aureus. The prepared inoculums were exposed for antibacterial activity with defined concentrations of the CdS nanoparticles $(20,40,60$ and $80 \mu \mathrm{g} / \mathrm{ml}$ ) The results were compared with commercially available antibiotics amoxicillin and ciprofloxicin. Triplicates were done for both the strains with defined concentration. The tubes were incubated at $37^{\circ} \mathrm{C}$ overnight. The turbidity was analyzed using spectroscopic analysis using UV-Vis Spectrophotometer (SHIMADZU-Model UV-1800 240 V) [12].

\section{Results}

\section{Culture and extraction of microbial biomass and synthesis of cadmium sulphide nanoparticles}

Bacillus licheniformis ATCC 12759 strain was incubated overnight and the culture was transferred to $50 \mathrm{ml}$ Falcon's centrifuge tube.Pure biomass of Bacillus licheniformis ATCC 12759 strain was used for the synthesis of CdS nanoparticles. Sodium sulphide and cadmium chloride were used for precipitation and the yellow precipitate confirmed the presence of nanoparticles in the mixture.

\section{Scanning electron microscopy}

SEM analysis revealed that the size of the thus CdS nanoparticles synthesized was between 15-20 nm (Figure 1).

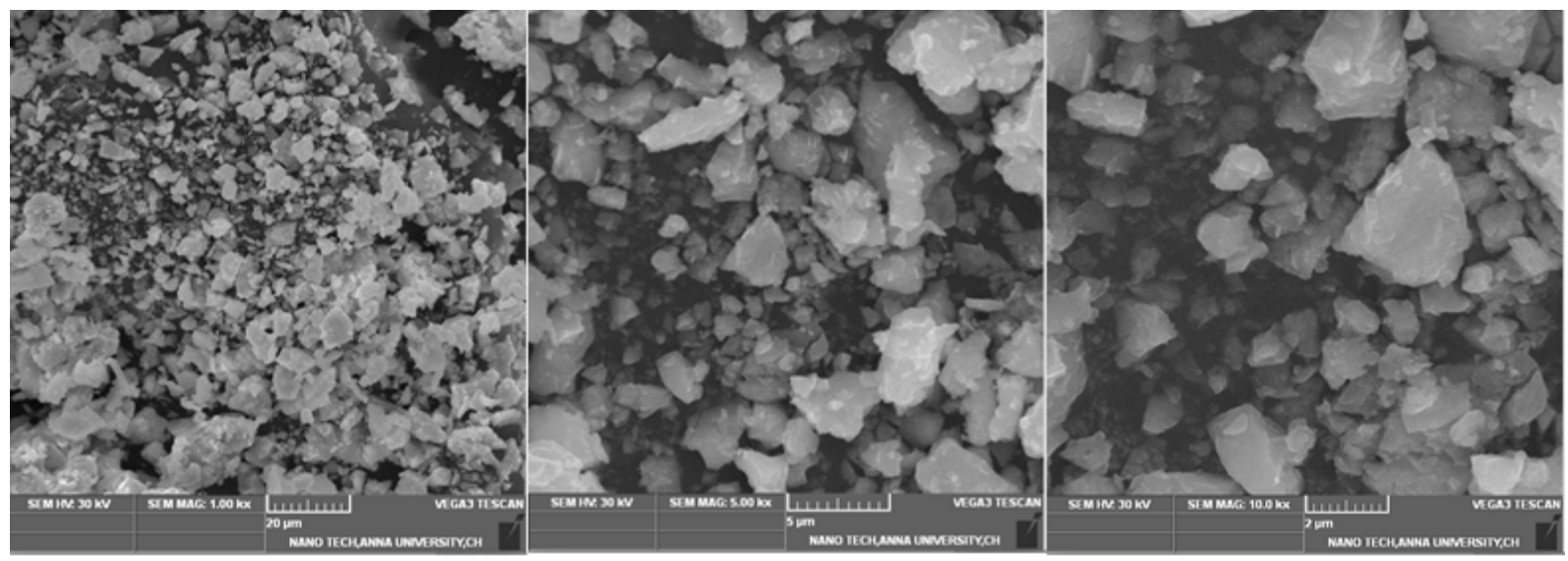

Figure 1. Scanning electron microscopy. 
Green nanotechnology in cadmium sulphide nanoparticles and understanding its toxicity and antimicrobial properties.

\section{In vivo toxicity assessment using Drosophila melanogaster}

Phenotypic analysis: Phenotypic analysis revealed that the $\mathrm{CdS}$ nanoparticles did not show any phenotypic changes till 60 $\mu \mathrm{g} / \mathrm{ml}$ concentration but a slight discoloration of body color for both the male and female adult flies at $80 \mu \mathrm{g} / \mathrm{ml}$ concentration was observed [13].

DNA fragmentation assay: DNA fragmentation analysis was documented using $2 \%$ agarose with different concentrations of the CdS nanoparticles. Analysis revealed that there was no distinct shearing in the defined concentration of $\mathrm{CdS}$ nanoparticles [14]

Antimicrobial sensitivity test by well diffusion method: The antimicrobial sensitivity test confirmed that the synthesized nanoparticles had good antimicrobial properties when tested against E.coli and Staphylococcus aureus as interpreted in Table 1 and shown in (Figure 2) respectively.

Table 1. Antibiotic Sensitivity Test.

\begin{tabular}{|c|c|c|c|c|c|}
\hline \multirow{2}{*}{ Microorganism and Zone of inhibition ( $\mathrm{mm}$ ) } & \multicolumn{5}{|c|}{ Concentration of Cadmium sulphide Np } \\
\hline & $20 \mu \mathrm{g} / \mathrm{ml}$ & $40 \mu \mathrm{g} / \mathrm{ml}$ & $60 \mu \mathrm{g} / \mathrm{ml}$ & $80 \mu \mathrm{g} / \mathrm{ml}$ & $40 \mu \mathrm{g} / \mathrm{ml}(\mathrm{PC})$ \\
\hline E. coli Zone of inhibition (mm) & 22 & 28 & 34 & 36 & 30 \\
\hline Staphylococcus aureus Zone of inhibition (mm) & 7 & 9 & 14 & 20 & 33 \\
\hline
\end{tabular}
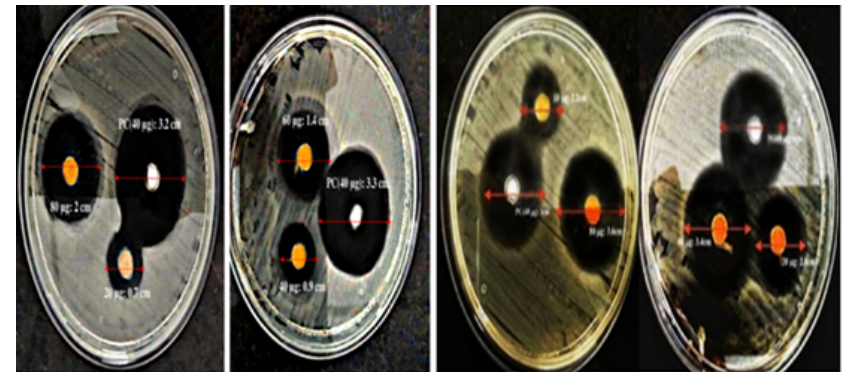

Anti microbial susceptibility test by macro-dilution method: Antimicrobial activity of the synthesized $\mathrm{CdS}$ nanoparticles was tested against Escherichia coli and Staphylococcus aureus. The results for the antibiotic susceptibility test were done using UV-Vis Spectrophotometer. The study revealed that the $\mathrm{CdS}$ nanoparticles showed significant antimicrobial activity as shown in Table 2 against the both the strains as in (Figures 3 and 4) respectively.

Figure 2. Well diffusion method for E coli and Staphyloccocus aureus (Zone of Clearance).

Table 2. Antibiotic Susceptibility by Macrodilution Method.

\begin{tabular}{ll}
\hline Concentration of CdS Nanoparticles $(\mathbf{m c q} / \mathbf{m l})$ & $\begin{array}{l}\text { OD for Staphylococcus aureus (Absorbance at } \\
\mathbf{6 0 0} \mathbf{n m})\end{array}$ \\
\hline 10 & $\begin{array}{l}\text { OD for E. coli (Absorbance } \\
\text { at } \mathbf{6 0 0} \mathbf{n m})\end{array}$ \\
\hline 20 & 0.853 \\
\hline 40 & 0.931 \\
\hline 80 & 0.902 \\
\hline Negative Control & 0.964 \\
\hline Positive Control & 1.623 \\
\hline
\end{tabular}




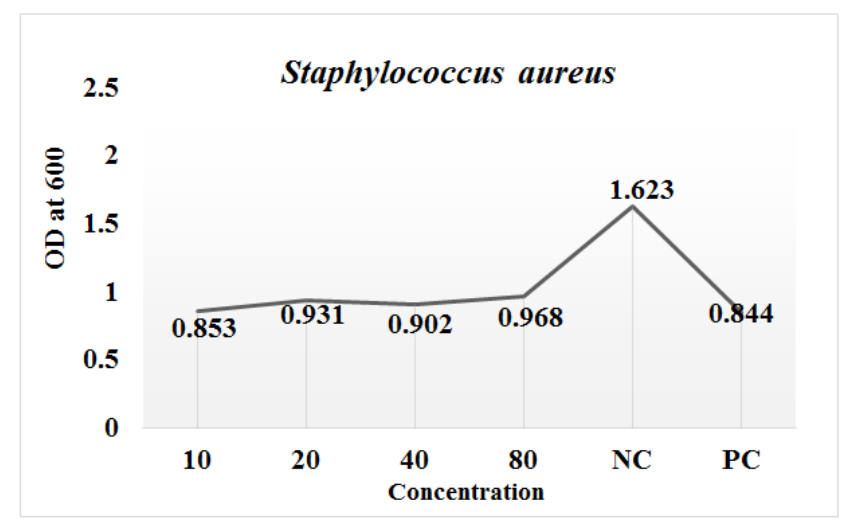

Figure 3. Antibiotic Susceptibility for Staphylococcus aureus by Macrodilution Method.

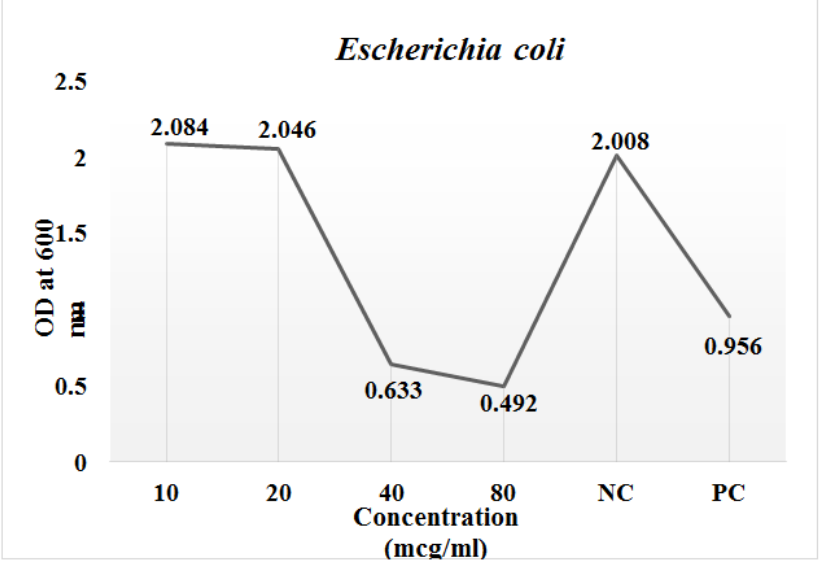

Figure 4. Antibiotic Susceptibility for Escherichia coli by Macrodilution Method.

\section{Discussion}

Biosynthesis of Cadmium sulphide nanoparticles using Bacillus licheniformis ATCC 12759 strain formed yellow colored mixture indicating the precipitation of cadmium sulphide nanoparticles. The lyophilized particles were characterized by Scanning Electron Microscopy (SEM). The SEM analysis confirmed the presence of CdS nanoclusters. These nanoclusters were further studied for genotoxicity assessment using Drosophila melanogaster as the model organism [15]. In vivo toxicity tests were done using Drosophila melanogaster to evaluate phenotypic and genotypic changes induced due to exposure of CdS nanoparticles. Results of the phenotypic analysis revealed that the CdS nanoparticles were non-toxic. The DNA of flies exposed to different concentrations of the nanoparticles was extracted, quantified and checked for purity using Nanodrop ND 1000 Spectrophotometer. The genotypic analysis revealed only mild shearing at higher concentrations of nanoparticles and no shearing at lower concentrations. The preliminary antimicrobial activity was tested by disc diffusion and macro dilution method against E.coli and staphylococcus aureus using different concentrations of the nanoparticles. The antimicrobial susceptibility test showed significant activity against the both the strains. CdS nanoparticles have been found to have beneficial applications furthermore possess physical properties such as high chemical and heat resistance, semiconductor, high opacity, high optical transmission and fluorescence that have successfully enabled its use as fluorescent biological labels, pigmentation agents and in solar cells. Biologically, CdS nanoparticles are stable and have proven to show antimicrobial properties [16]. Hence, CdS nanoparticles can be efficiently brought into application for its antimicrobial ability on analyzing its activity as per the quantity stated as 'Permissible levels in adults' formulated by the World Health Organization (WHO) and US Food and Drug Administration (US-FDA) [17,18]. It is essential to evaluate any further toxicological risk of the formulated nanomaterial before it is being commercially used.

\section{Conclusion}

The present study revealed that the $\mathrm{CdS}$ nanoparticles synthesized by the green, eco-friendly method was stable and characterized to determine the size. The toxicity studies revealed that the $\mathrm{CdS}$ nanoparticles were not toxic. The antimicrobial activity showed a significant effect on both the gram positive and gram negative bacteria.

\section{Acknowledgement}

We would like to thank the Department of Biomedical Sciences, Sri Ramachandra Institute of Higher Education and Research for the facilities provided for the conduct of the study. We take this opportunity to thank the Centre for Nanoscience and Technology, Anna University, Chennai, India for allowing us to use their facility for the SEM analysis.

\section{REFERENCES}

1. Liu J, Qiao SZ, Hu QH, Lu GQ. Magnetic nanocomposites with mesoporous structures: synthesis and applications. Small 2011; 7: 425-243.

2. Luechinger NA, Grass RN, Athanassiou EK, Stark WJ. Bottom-up fabrication of metal/metal nanocomposites from nanoparticles of immiscible metals. Chem Mater 2010; 22: 155-160.

3. Tiwari DK, Behari J, Sen P. Time and dose-dependent antimicrobial potential of Ag nanoparticles synthesized by top-down approach. Current Science 2008; 95: 647-655.

4. Mohanpuria P, Rana NK, Yadav SK. Biosynthesis of nanoparticles: technological concepts and future applications. Nano Res 2008; 10: 507-517.

5. Ayesha Arshad. Bacterial Synthesis and Applications of Nanoparticles. Nano Sci Nano Technol 2017; 11: 119.

6. Nida Qutub. Cadmium sulphide nanoparticles. Ph.D.Thesis 2013.

7. Jiang W, Singhal A, Zheng J, Wang C, Chan CW, Optimizing the Synthesis of Red-to Near-IR-Emitting CdSCapped CdTexSe1-x Alloyed Quantum Dots for Biomedical Imaging. Chem Mate 2006; 18: 4845--4854. 
properties.

8. Kumar A, Singh S, Kumar D. Evaluation of Antimicrobial Potential of Cadmium Sulphide Nanoparticles against Bacterial Pathogens. Int J Pharm Sc Re 2014; 24: 202-207.

9. Jing Z, Tan L, Li F, Wang J, Fu Y, Li Q. Photocatalytic and Antibacterial activities of $\mathrm{CdS}$ nanoparticles prepared by solvothermal method. Ind J Chem 2013; 52: 55-62.

10. Barman J, Sultana F. Synthesis of mix Zinc Oxide and Cadmium Sulphide Nanoparticles, Optoelectronic and antimicrobial activity and application in Water treatment. IOSR-JAP 2016; 8: 46-51.

11. Tripathi RM. Mechanistic aspects of biogenic synthesis of CdS Nanoparticles using Bacillus Licheniformis. Adv Nat Sci Nanosci Nanotechnol 2014; 5: 25006.

12. Shivashankarappa A, Sanjay KR. Study on Biological Synthesis of Cadmium Sulphide Nanoparticles by Bacillus licheniformis and its Antimicrobial Properties against Food Borne Pathogens. Nanosci Nanotechnol Res 2015; 3: 6-15.

13. Chitwood LA. Tube Dilution Antimicrobial Susceptibility Testing: Efficacy of a Microtechnique Applicable to Diagnostic Laboratories. Appli Microbiol 1969; 17: 707-709.

14. Haltalin KC, Markley AH, Woodman E. Agar Plate Dilution Method for Routine Antibiotic Susceptibility Testing in a Hospital Laboratory Ame J Clin Pathol 1973; 60: 384-394.
15. Guzmán MG, Dille J, Godet S. Synthesis of silver nanoparticles by chemical reduction method and their antibacterial activity. Int J Chem Biomol Eng 2009; 2: 3.

16. Salata OV. Applications of nanoparticles in biology and medicine. J Nanobiotech 2004; 2: 3.

17. Cadmium in Drinking-water: Background document for development of WHO Guidelines for Drinking-water Quality 2017.

18. Wu Q, Huang L,Li Z, An W, Liu D, Lin J, Tian L, Wang X, Liu B, Qi W, Wu W. The Potential Application of Raw Cadmium Sulfide Nanoparticles as CT Photographic Developer Nanoscale Res Lett 2016; 11: 232.

\section{*Correspondence to}

Sumitha R

Department of Biomedical Sciences

Sri Ramachandra Institute of Higher Education and Research

Chennai

India 